PROCEEDINGS OF THE

AMERICAN MATHEMATICAL SOCIETY

Volume 128, Number 8, Pages 2381-2391

S 0002-9939(00)05648-3

Article electronically published on February 21, 2000

\title{
BIVARIATE VERSION OF THE HAHN-SONINE THEOREM
}

\author{
JEONGKEUN LEE
}

(Communicated by Hal L. Smith)

\begin{abstract}
We consider orthogonal polynomials in two variables whose derivatives with respect to $x$ are orthogonal. We show that they satisfy a system of partial differential equations of the form

$$
\alpha(x, y) \partial_{x}^{2} \vec{U}_{n}+\beta(x, y) \partial_{x} \vec{U}_{n}=\Lambda_{n} \vec{U}_{n},
$$

where $\operatorname{deg} \alpha \leq 2, \operatorname{deg} \beta \leq 1, \vec{U}_{n}=\left(U_{n 0}, U_{n-1,1}, \cdots, U_{0 n}\right)$ is a vector of polynomials in $x$ and $y$ for $n \geq 0$, and $\Lambda_{n}$ is an eigenvalue matrix of order $(n+1) \times(n+1)$ for $n \geq 0$. Also we obtain several characterizations for these polynomials. Finally, we point out that our results are able to cover more examples than Bertran's.
\end{abstract}

\section{INTRODUCTION}

We are concerned with the problem of characterizing orthogonal polynomials in two variables whose partial derivatives with respect to $x$ are also orthogonal. W. Hahn [5] solved the univariate version of this problem partially. He showed that the only positive-definite orthogonal polynomials whose derivatives also form positive-definite orthogonal polynomials are Jacobi, Laguerre or Hermite polynomials. Later H. L. Krall [10] extended Hahn's results to the quasi-definite case and showed that the only orthogonal polynomials whose derivatives also form orthogonal polynomials are the classical orthogonal polynomials of Jacobi, Laguerre, Hermite and Bessel. In addition to the work of Bochner and Krall (see also [14), there are many characterizations for the classical orthogonal polynomials (see [1, [3], 13, [17]). For example, the following statements are equivalent:

Let $\left\{P_{n}(x)\right\}_{n=0}^{\infty}$ be an orthogonal polynomial set (i.e., $\operatorname{deg} P_{n}=n$ for all $n \geq 0$ ).

(i) For each $n \geq 0, y=P_{n}(x)$ satisfies a second order ordinary differential equation

$$
\alpha(x) y^{\prime \prime}(x)+\beta(x) y^{\prime}(x)=\lambda_{n} y(x),
$$

for some $\alpha(x)=a x^{2}+b x+c \neq 0$ and $\beta(x)=d x+e$, where $\lambda_{n}=a n(n-1)+$ $d n \neq 0$ if $n \geq 1$.

(ii) Up to a complex linear change of variable, $\left\{P_{n}(x)\right\}_{n=0}^{\infty}$ is one of the following sets of orthogonal polynomials:

(a) Jacobi polynomials $\left\{P_{n}^{(\alpha, \beta)}(x)\right\}_{n=0}^{\infty}$ for some $\alpha, \beta, \alpha+\beta+1 \notin\{-1,-2, \cdots\}$,

(b) Laguerre polynomials $\left\{L_{n}^{(\alpha)}(x)\right\}_{n=0}^{\infty}$ for some $\alpha \notin\{-1,-2, \cdots\}$,

Received by the editors September 19, 1998.

1991 Mathematics Subject Classification. Primary 33C50, 35P99.

Key words and phrases. Orthogonal polynomials in two variables, Hahn-Sonine theorem. 
(c) Hermite polynomials $\left\{H_{n}(x)\right\}_{n=0}^{\infty}$,

(d) Bessel polynomials $\left\{B_{n}^{(\alpha, \beta)}(x)\right\}_{n=0}^{\infty}$ for some $\alpha \notin\{0,-1,-2, \cdots\}$ and $\beta \neq 0$.

(iii) There exists a nonzero orthogonalizing moment functional $\sigma$ for $\left\{P_{n}(x)\right\}_{n=0}^{\infty}$ such that

$$
(\alpha(x) \sigma)^{\prime}=\beta(x) \sigma
$$

for some polynomial $\alpha(x)$ of degree $\leq 2$ and $\beta(x)$ of degree $\leq 1$.

(iv) $\left\{P_{n}^{\prime}(x)\right\}_{n=1}^{\infty}$ is weakly orthogonal; i.e., there exists a nonzero moment functional $\tau$ such that

$$
\left\langle\tau, P_{n}^{\prime}(x) P_{m}^{\prime}(x)\right\rangle=0 \quad \text { if } n \neq m .
$$

(v) $\left\{P_{n}(x)\right\}_{n=0}^{\infty}$ satisfies a difference-differential equation of the form

$$
\alpha(x) P_{n}^{\prime}(x)=\left(r_{n} x+s_{n}\right) P_{n}(x)+t_{n} P_{n-1}(x)
$$

for some nonzero polynomial $\alpha(x)$ of degree $\leq 2$ and constants $r_{n}, s_{n}$ and $t_{n}$.

The study of classical orthogonal polynomials in one variable leads us to consider the relationship between orthogonal polynomials in two variables and a second order partial differential equation of the form

$$
A u_{x x}+2 B u_{x y}+C u_{y y}+D u_{x}+E u_{y}=\lambda_{n} u,
$$

where $A(x, y), B(x, y)$ and $C(x, y)$ are polynomials of degree $\leq 2, D(x, y)$ and $E(x, y)$ are polynomials of degree $\leq 1$, and $\lambda_{n}$ is the eigenvalue parameter. The important work in this direction was done by Krall and Sheffer in 1967 [11. But there have been only a few results in this area since Krall and Sheffer. One of the possible reasons lies in the fact that the general theory of multivariate orthogonal polynomials was developed only very recently. The matrix-vector notation has played an essential role in the recent development of the general theory. It was introduced by Kowalski ( 8 , 9 $)$. He characterized the orthogonality of polynomials in several variables by the recurrence relations with matrix coefficients. $\mathrm{Xu}[18]$ gave another formulation of the recurrence relations and gave a simple proof of Kowalski's theorem.

Owing to the three term recurrence relations with matrix coefficients, we [6] obtained a characterization for orthogonal polynomials in two variables which satisfy the partial differential equation of the form (1.5). In fact, we generalized the property (v) to orthogonal polynomials in two variables.

In this paper, we generalize the Hahn-Sonine theorem to bivariate orthogonal polynomials and obtain characterizations analogous to the classical orthogonal polynomials. In particular, we show that a generalization of (iv) does not produce a second order partial differential equation of the form (1.5) but does produce a second order partial differential equation with two-parameter eigenvalues. Finally, we show that our results can cover more examples which cannot be covered by Bertran's (see Examples 3.1 and 3.3.

\section{MAin CONTENTS}

The set of all polynomials in two variables will be denoted by $\mathcal{P}$. By a polynomial system (PS), we mean a sequence of polynomials $\left\{\phi_{m n}(x, y)\right\}_{m, n=0}^{\infty}$ such that $\operatorname{deg} \phi_{m n}=m+n$ for all $m, n \geq 0$ and $\left\{\phi_{n-j, j}\right\}_{j=0}^{n}$ is linearly independent modulo polynomials of degree $\leq n-1$. For brevity, we denote $\left\{\phi_{n-j, j}\right\}_{j=0}^{n}$ by an $(n+1) \times 1$ 
matrix $\Phi_{n}$ and a $\operatorname{PS}\left\{\phi_{m n}(x, y)\right\}_{m, n=0}^{\infty}$ by $\left\{\Phi_{n}\right\}_{n=0}^{\infty}$. A PS $\left\{\Phi_{n}\right\}_{n=0}^{\infty}$ is said to be monic if $\phi_{m n}(x, y)=x^{m} y^{n}+$ lower degree terms.

We call any linear functional on $\mathcal{P}$ a moment functional. We denote the action of a moment functional $\sigma$ on polynomial $\pi$ by $\langle\sigma, \pi\rangle$. Similarly, for a matrix $Q=\left(Q_{i, j}\right)$ with $Q_{i, j}$ being a polynomial, $\langle\sigma, Q\rangle$ is defined to be the matrix $\left(\left\langle\sigma, Q_{i, j}\right\rangle\right)$. We see that $\left\langle\sigma, A B^{T}\right\rangle=\left\langle\sigma, B A^{T}\right\rangle^{T}$ for any column vectors $A$ and $B$ of polynomials.

For a moment functional $\sigma$, we define the partial derivatives of $\sigma$ by the formulas

$$
\left\langle\partial_{x} \sigma, \phi\right\rangle=-\left\langle\sigma, \partial_{x} \phi\right\rangle, \quad\left\langle\partial_{y} \sigma, \phi\right\rangle=-\left\langle\sigma, \partial_{y} \phi\right\rangle, \quad \phi \in \mathcal{P}
$$

and define the multiplication on $\sigma$ by a polynomial $\psi$ through the formula

$$
\langle\psi \sigma, \phi\rangle=\langle\sigma, \psi \phi\rangle, \quad \phi \in \mathcal{P} .
$$

Definition 2.1. A PS $\left\{\Phi_{n}\right\}_{n=0}^{\infty}$ is called an orthogonal basis (OB) relative to $\sigma$ if there is a nonzero moment functional $\sigma$ such that

$$
\left\langle\sigma, \phi_{m n} \phi_{k l}\right\rangle=0 \quad \text { if } m+n \neq k+l .
$$

We say that $\left\{\Phi_{n}\right\}_{n=0}^{\infty}$ is a weak orthogonal polynomial system (WOPS) relative to $\sigma$ if

$$
\left\langle\sigma, \phi_{m n} \phi_{k l}\right\rangle=K_{m n} \delta_{m k} \delta_{n l}
$$

If $K_{m n} \neq 0$ for each $m, n \geq 0$, then we say that $\left\{\Phi_{n}\right\}_{n=0}^{\infty}$ is an orthogonal polynomial set (OPS) relative to $\sigma$.

It is obvious that there is an OB relative to $\sigma$ if and only if there is a WOPS relative to $\sigma$.

Definition 2.2. A moment functional $\sigma$ is quasi-definite (resp., weakly quasidefinite) if there is an OPS (resp., a WOPS) relative to $\sigma$.

From Definition 2.1, we see that a PS $\left\{\Phi_{n}\right\}_{n=0}^{\infty}$ is a WOPS (resp., an OPS) relative to $\sigma$ if and only if $\left\langle\sigma, \Phi_{m} \Phi_{n}^{T}\right\rangle=H_{n} \delta_{m n}$ and $H_{n}:=\left\langle\sigma, \Phi_{n} \Phi_{n}^{T}\right\rangle$ is a diagonal (resp., a nonsingular diagonal) matrix.

For any $\operatorname{PS}\left\{\Phi_{n}\right\}_{n=0}^{\infty}$, there is a unique moment functional $\sigma$, which is called the canonical moment functional of $\left\{\Phi_{n}\right\}_{n=0}^{\infty}$, defined by the conditions

$$
\langle\sigma, 1\rangle=1, \quad\left\langle\sigma, \phi_{m n}\right\rangle=0, m+n \geq 1 .
$$

Note that if a $\operatorname{PS}\left\{\Phi_{n}\right\}_{n=0}^{\infty}$ is an OB relative to $\sigma$, then $\sigma$ is a constant multiple of canonical moment functional of $\left\{\Phi_{n}\right\}_{n=0}^{\infty}$.

Theorem 2.1 ([6], 11]). For any moment functional $\sigma$, the following statements are equivalent:

(i) $\sigma$ is quasi-definite.

(ii) There is a unique monic $O B$ relative to $\sigma$.

(iii) There is a monic $O B\left\{\mathbb{P}_{n}\right\}_{n=0}^{\infty}$ such that $H_{n}:=\left\langle\sigma, \mathbb{P}_{n} \mathbb{P}_{n}^{T}\right\rangle$ is nonsingular for all $n \geq 0$.

Lemma 2.2 ([6]). Let $\sigma$ and $\tau$ be moment functionals and $\phi$ be a polynomial. Then

(i) $\sigma=0$ if and only if $\partial_{x} \sigma=\partial_{y} \sigma=0$.

(ii) Let $\left\{\Phi_{n}\right\}_{n=0}^{\infty}$ be an $O B$ relative to a quasi-definite moment functional $\sigma$. Then

(a) $\phi \sigma=0$ if and only if $\phi \equiv 0$;

(b) $\left\langle\tau, \Phi_{n}\right\rangle=0$ for $n>k$ if and only if $\tau=\psi \sigma$ for some polynomial $\psi$ of degree $\leq k$. 
Theorem 2.3 (Favard's Theorem [18]). Let $\left\{\Phi_{n}\right\}_{n=0}^{\infty}$ be a PS. Then the following statements are equivalent:

(i) $\left\{\Phi_{n}\right\}_{n=0}^{\infty}$ is an $O B$ relative to a quasi-definite moment functional $\sigma$.

(ii) For $n \geq 0$ and $i=1,2$, there are matrices $A_{n i}$ of order $(n+1) \times(n+2), B_{n i}$ of order $(n+1) \times(n+1)$, and $C_{n i}$ of order $(n+1) \times n$ such that

(a) $x_{i} \Phi_{n}=A_{n i} \Phi_{n+1}+B_{n i} \Phi_{n}+C_{n i} \Phi_{n-1}$;

(b) $\operatorname{rank} C_{n}=n+1$, where $C_{n}=\left(C_{n 1}, C_{n 2}\right)$.

Now we are ready to state our main results.

Theorem 2.4. Let $\left\{\mathbb{P}_{n}\right\}_{n=0}^{\infty}$ be a monic OB relative to a quasi-definite moment functional $\sigma$. Assume that $\partial_{x} P_{0 n} \equiv 0$ (resp., $\partial_{y} P_{n 0} \equiv 0$ ) for all $n \geq 0$. Then the following statements are equivalent:

(i) $\sigma$ satisfies the functional equation of the form

$$
(\alpha(x, y) \sigma)_{x}=\beta(x, y) \sigma\left(\text { resp. },(\bar{\alpha}(x, y) \sigma)_{y}=\bar{\beta}(x, y) \sigma\right),
$$

where $\alpha(x, y)$ (resp., $\bar{\alpha}(x, y))$ is a polynomial of degree $\leq 2$ and $\beta(x, y)$ (resp., $\bar{\beta}(x, y))$ is a polynomial of degree $\leq 1$.

(ii) $\left\{\mathbb{P}_{n}\right\}_{n=0}^{\infty}$ satisfy a system of equations of the form

$$
\alpha(x, y) \partial_{x}^{2} \vec{U}_{n}+\beta(x, y) \partial_{x} \vec{U}_{n}=\Lambda_{n} \vec{U}_{n}\left(\text { resp. }, \bar{\alpha}(x, y) \partial_{y}^{2} \vec{U}_{n}+\bar{\beta}(x, y) \partial_{y} \vec{U}_{n}=\Gamma_{n} \vec{U}_{n}\right),
$$

where $\alpha(x, y)$ (resp., $\bar{\alpha}(x, y))$ is a polynomial of degree $\leq 2$ and $\beta(x, y)$ (resp., $\bar{\beta}(x, y))$ is a polynomial of degree $\leq 1, \Lambda_{n}$ (resp., $\left.\Gamma_{n}\right)$ is a constant matrix of order $(n+1) \times(n+1)$ and $\vec{U}_{n}=\left(U_{n 0}, U_{n-1,1}, \cdots, U_{0 n}\right)^{T}$ is a vector of polynomials for each $n \geq 0$.

(iii) $\left\{\mathbb{P}_{n}^{(x)}\right\}_{n=0}^{\infty}$ (resp., $\left\{\mathbb{P}_{n}^{(y)}\right\}_{n=0}^{\infty}$ ) is a monic OB, where

$$
\begin{aligned}
& \mathbb{P}_{n}^{(x)}=\left(P_{n 0}^{(x)}, P_{n-1,1}^{(x)}, \cdots, P_{0 n}^{(x)}\right)^{T}, P_{n-k, k}^{(x)}=\frac{1}{n+1-k} \partial_{x} P_{n+1-k, k} \quad \text { for } 0 \leq k \leq n, \\
& \mathbb{P}_{n}^{(y)}=\left(P_{n 0}^{(y)}, P_{n-1,1}^{(y)}, \cdots, P_{0 n}^{(y)}\right)^{T}, P_{n-k, k}^{(y)}=\frac{1}{k+1} \partial_{y} P_{n-k, k+1} \text { for } 0 \leq k \leq n .
\end{aligned}
$$

Proof. We prove the case that $\partial_{x} P_{0 n} \equiv 0$ only since the other case can be proved similarly.

$(i) \Longrightarrow(i i)$ : Since $\alpha(x, y) \partial_{x}^{2} \mathbb{P}_{n}+\beta(x, y) \partial_{x} \mathbb{P}_{n}$ is a vector of polynomials of degree $\leq n$, there are matrices $A_{j}^{n}$ of order $(n+1) \times(j+1)(j=0,1, \cdots, n)$ such that

$$
\alpha(x, y) \partial_{x}^{2} \mathbb{P}_{n}+\beta(x, y) \partial_{x} \mathbb{P}_{n}=\sum_{j=0}^{n} A_{j}^{n} \mathbb{P}_{j}
$$

Then we have for $0 \leq k \leq n-1$

$$
\begin{aligned}
& A_{k}^{n} H_{k}=A_{k}^{n}\left\langle\sigma, \mathbb{P}_{k} \mathbb{P}_{k}^{T}\right\rangle=\left\langle\sigma, \sum_{j=0}^{n} A_{j}^{n} \mathbb{P}_{j} \mathbb{P}_{k}^{T}\right\rangle=\left\langle\sigma,\left(\alpha \partial_{x}^{2} \mathbb{P}_{n}+\beta \partial_{x} \mathbb{P}_{n}\right) \mathbb{P}_{k}^{T}\right\rangle \\
& =\left\langle\alpha \sigma, \partial_{x}\left(\partial_{x} \mathbb{P}_{n} \mathbb{P}_{k}^{T}\right)-\partial_{x} \mathbb{P}_{n} \partial_{x} \mathbb{P}_{k}^{T}\right\rangle+\left\langle\beta \sigma, \partial_{x} \mathbb{P}_{n} \mathbb{P}_{k}^{T}\right\rangle=-\left\langle\alpha \sigma, \partial_{x} \mathbb{P}_{n} \partial_{x} \mathbb{P}_{k}^{T}\right\rangle \\
& =-\left\langle\alpha \sigma, \partial_{x}\left(\mathbb{P}_{n} \mathbb{P}_{k}^{T}\right)-\mathbb{P}_{n} \partial_{x}^{2} \mathbb{P}_{k}^{T}\right\rangle=\left\langle\sigma, \mathbb{P}_{n}\left(\alpha \partial_{x}^{2} \mathbb{P}_{k}+\beta \partial_{x} \mathbb{P}_{k}\right)^{T}\right\rangle=0
\end{aligned}
$$

since $\operatorname{deg}\left(\alpha \partial_{x}^{2} \mathbb{P}_{k}+\beta \partial_{x} \mathbb{P}_{k}\right) \leq 2$. Hence we have $A_{k}^{n}=0$ for all $0 \leq k \leq n-1$. By comparing the coefficients of the highest degree terms in both sides in (2.3), we obtain (ii). 
$(i i) \Longrightarrow($ iii $)$ : Let $\mathbb{P}_{n}^{(x)}=\left(\partial_{x} P_{n+1,0}, \partial_{x} P_{n, 1}, \cdots, \partial_{x} P_{1, n}\right)^{T}$. Then for $m \neq n$, we have

$$
\begin{aligned}
& \left\langle\alpha \sigma, \partial_{x} \mathbb{P}_{n+1} \partial_{x} \mathbb{P}_{m+1}^{T}\right\rangle=\left\langle\alpha \sigma, \partial_{x}\left(\mathbb{P}_{n+1} \partial_{x} \mathbb{P}_{m+1}^{T}\right)-\mathbb{P}_{n+1} \partial_{x}^{2} \mathbb{P}_{m+1}^{T}\right\rangle \\
& =-\left\langle\sigma, \mathbb{P}_{n+1}\left(\alpha \partial_{x}^{2} \mathbb{P}_{m}+\beta \partial_{x} \mathbb{P}_{m}\right)^{T}\right\rangle=-\left\langle\sigma, \mathbb{P}_{n+1} \mathbb{P}_{m+1}^{T}\right\rangle \Lambda_{m+1}^{T}=0
\end{aligned}
$$

since $\left\{\mathbb{P}_{n}\right\}_{n=0}^{\infty}$ is an OB $\sigma$. Thus $\left\{\mathbb{P}_{n}^{(x)}\right\}_{n=0}^{\infty}$ is a monic OB relative to $\left.\alpha \sigma\right)$ since $\left\langle\alpha \sigma, \partial_{x} \mathbb{P}_{n+1} \partial_{x} \mathbb{P}_{m+1}\right\rangle=0$ implies $\left\langle\alpha \sigma, \mathbb{P}_{n}^{(x)} \mathbb{P}_{m}^{(x) T}\right\rangle=0$.

$($ iii $) \Longrightarrow(i)$ : Let $\tau$ be the canonical moment functional of $\left\{\mathbb{P}_{n}^{(x)}\right\}_{n=0}^{\infty}$. Note that $\left\langle\tau, \mathbb{P}_{m}^{(x)} \mathbb{P}_{n}^{(x) T}\right\rangle=0$ implies that $\left\langle\tau, \partial_{x} \mathbb{P}_{m+1} \partial_{x} \mathbb{P}_{n+1}^{T}\right\rangle=0$

If we take $m=0$, then for $n>0$ we have

$$
\left\langle\tau, \partial_{x} \mathbb{P}_{n+1}\right\rangle=-\left\langle\tau_{x}, \mathbb{P}_{n+1}\right\rangle=0
$$

Hence by Lemma 2.2 , there is a polynomial $\beta$ of degree $\leq 1$ such that

$$
\tau_{x}=\beta \sigma .
$$

If we take $m=1$, then we have for $n>1$

$$
\left\langle\tau, \partial_{x} P_{20} \partial_{x} \mathbb{P}_{n+1}^{T}\right\rangle=0, \quad\left\langle\tau, \partial_{x} P_{11} \partial_{x} \mathbb{P}_{n+1}^{T}\right\rangle=0 .
$$

Hence by Lemma 2.2, there are polynomials $\alpha_{0}(x, y), \alpha_{1}(x, y)$ of degree $\leq 2$ such that

$$
\left(\partial_{x} P_{20} \tau\right)_{x}=\alpha_{0} \sigma, \quad\left(\partial_{x} P_{11} \tau\right)_{x}=\alpha_{1} \sigma .
$$

Thus we have $\tau=\frac{1}{2}\left(\alpha_{0}-\beta \partial_{x} P_{20}\right) \sigma$ and $\frac{1}{2}\left[\left(\alpha_{0}-\beta \partial_{x} P_{20}\right) \sigma\right]_{x}=\beta \sigma$. Since $\alpha_{0}-$ $\beta \partial_{x} P_{20}$ is a nonzero polynomial of degree $\leq 2$, (i) is proved.

Remark 2.1. The condition that $\partial_{x} P_{0 n}=0$ for all $n \geq 0$ is not too restrictive. In fact, all weak orthogonal polynomials found by Krall and Sheffer [11] satisfy this condition.

Theorem 2.5. If $\Lambda_{n}$ (resp., $\Gamma_{n}$ ) in (2.2) is diagonalizable for all $n \geq 0$, there is an $O B\left\{\Phi_{n}\right\}_{n=0}^{\infty}$ relative to $\sigma$ such that $\left\{\Phi_{n}\right\}_{n=0}^{\infty}$ satisfy a partial differential equation with two-parameter eigenvalues

$\alpha(x, y) \partial_{x}^{2} u+\beta(x, y) \partial_{x} u=\lambda_{n-k, k} u, \quad\left(\right.$ resp., $\left.\bar{\alpha}(x, y) \partial_{y}^{2} u+\bar{\beta}(x, y) \partial_{y} u=\gamma_{n-k, k} u\right)$, where $\lambda_{n-k, k}\left(\right.$ resp., $\left.\gamma_{n-k, k}\right)$ are the eigenvalues of $\Lambda_{n}\left(\right.$ resp., $\left.\Gamma_{n}\right)$ for $0 \leq k \leq n$.

Proof. Let $A_{n}$ be a nonsingular matrix of order $(n+1) \times(n+1)$ such that

$$
A_{n}^{-1} \Lambda_{n} A_{n}=D_{n}
$$

where $D_{n}$ is a diagonal matrix $D_{n}=\operatorname{Diag}\left(\lambda_{n 0}, \lambda_{n-1,1}, \cdots, \lambda_{0 n}\right)$. Define a PS $\left\{\Phi_{n}\right\}_{n=0}^{\infty}$ by $\Phi_{n}=A_{n}^{-1} \mathbb{P}_{n}$. Then $\left\{\Phi_{n}\right\}_{n=0}^{\infty}$ satisfy a partial differential equation (2.4). Thus the theorem is proved.

We can obtain similar results for $\Gamma_{n}$.

Theorem 2.6. Let $\left\{\mathbb{P}_{n}\right\}_{n=0}^{\infty}$ be a monic $O B$ relative to a quasi-definite moment functional $\sigma$. Suppose that $\partial_{y} P_{n 0}=\partial_{x} P_{0 n}=0$ for all $n \geq 0$. If $\left\{\mathbb{P}_{n}^{(x)}\right\}_{n=0}^{\infty}$ and $\left\{\mathbb{P}_{n}^{(y)}\right\}_{n=0}^{\infty}$ are OB's relative to quasi-definite moment functionals, then $\left\{\mathbb{P}_{n}\right\}_{n=0}^{\infty}$ satisfies a second order partial differential equation of the form

$$
A(x, y) \partial_{x}^{2} \vec{U}_{n}+C(x, y) \partial_{y}^{2} \vec{U}_{n}+D(x, y) \partial_{x} \vec{U}_{n}+E(x, y) \partial_{y} \vec{U}_{n}=\Omega_{n} \vec{U}_{n},
$$


where $\operatorname{deg} A, \operatorname{deg} B \leq 2$ and $\operatorname{deg} D, \operatorname{deg} E \leq 1$ and $\Omega_{n}$ are eigenvalue matrices of order of $(n+1) \times(n+1)$.

Proof. The theorem is proved by applying Theorem 2.4 repeatedly.

Theorem 2.7. If each $\Omega_{n}$ in 2.5) is diagonalizable for each $n \geq 0$, then there is an $O B\left\{\Phi_{n}\right\}_{n=0}^{\infty}$ satisfying a second order partial differential equation with twoparameter eigenvalues

$$
A(x, y) u_{x x}+C(x, y) u_{y y}+D(x, y) u_{x}+E(x, y) u_{y}=\omega_{n-k, k} u,
$$

where $\omega_{n-k, k}(0 \leq k \leq n)$ are eigenvalues of $\Omega_{n}$.

Proof. The theorem is proved in the same way as in Theorem 2.5.

Remark 2.2. 1. The conditions that $\partial_{y} P_{n 0}=\partial_{x} P_{0 n}=0$ for all $n \geq 0$ hold for many OPS's. For OPS's satisfying a second order partial differential equation of the form (1.5), these conditions are equivalent to $A_{y}=C_{x}=0$. See [6] for the details and their properties.

2. In fact, $\Omega_{n}=\Lambda_{n}+\Gamma_{n}$, where $\Lambda_{n}$ and $\Gamma_{n}$ are given in (2.2).

\section{EXAMPles AND BERTRAN's WORK}

Let $\left\{\Phi_{n}\right\}_{n=0}^{\infty}$ be an OB relative to a quasi-definite moment functional $\sigma$ satisfying a second order partial differential equation of the form

$$
A u_{x x}+2 B u_{x y}+C u_{y y}+D u_{x}+E u_{y}=\lambda_{n} u .
$$

If we write $\left\{\Phi_{n}\right\}_{n=0}^{\infty}$ by

$$
\phi_{n-j, j}(x, y)=\sum_{k=0}^{n} a_{j k}^{n} x^{n-k} y^{k}+\text { lower terms } \quad(j=0,1, \cdots, n),
$$

then the monic PS $\left\{\mathbb{P}_{n}\right\}_{n=0}^{\infty}$, defined by

$$
\mathbb{P}_{n}=A_{n}^{-1} \Phi_{n}, \quad \text { where } A_{n}=\left(a_{j k}^{n}\right)_{j, k=0}^{n}(n \geq 0),
$$

also satisfies the partial differential equation (3.1). We call this PS $\left\{\mathbb{P}_{n}\right\}_{n=0}^{\infty}$ the normalization of $\left\{\Phi_{n}\right\}_{n=0}^{\infty}$. From now on, $\left\{\mathbb{P}_{n}\right\}_{n=0}^{\infty}$ will denote the normalization of an $\mathrm{OB}\left\{\Phi_{n}\right\}_{n=0}^{\infty}$ relative to a quasi-definite moment functional $\sigma$.

The partial differential equation (3.1) was introduced and investigated by Krall and Sheffer. Then it is well known (see [6, 11]) that $\sigma$ satisfies the following functional equations, called the moment equations,

$$
\left\{\begin{array}{l}
(A \sigma)_{x}+(B \sigma)_{y}-D \sigma=0 \\
(B \sigma)_{x}+(C \sigma)_{y}-E \sigma=0 .
\end{array}\right.
$$

Solving the moment equations (3.2) for $\sigma_{x}$ and $\sigma_{y}$, we have

$$
\left\{\begin{array}{l}
\left(\left(B^{2}-A C\right) \sigma\right)_{x}=\left[\left(B^{2}-A C\right)_{x}+\left(A_{x}+B_{y}-D\right) C-\left(B_{x}+C_{y}-E\right) B\right] \sigma, \\
\left(\left(B^{2}-A C\right) \sigma\right)_{y}=\left[\left(B^{2}-A C\right)_{y}-\left(A_{x}+B_{y}-D\right) B+\left(B_{x}+C_{y}-E\right) A\right] \sigma .
\end{array}\right.
$$

Note that $\operatorname{deg}\left(A C-B^{2}\right) \leq 3$ and the coefficients in (3.3) is of degree $\leq 2$.

Bertran [2] studied some properties of orthogonal polynomials in $\nu$-variables obtained by orthogonalizing an ordered basis of monomials. He derived a system of partial differential equations of the form (2.4) for these orthogonal polynomials 
under the strong conditions that the weight function $w(\mathbf{x})$ satisfies the system of first order partial differential equations with boundary conditions

$$
\alpha_{i} \partial_{x_{i}} w=\beta_{i} w, \quad \alpha_{i} w=0 \text { on } \partial \mathbf{R} \quad(i=1,2, \cdots, \nu),
$$

where $\operatorname{deg} \alpha_{i} \leq 2, \operatorname{deg} \beta_{i} \leq 1$ and $\mathbf{R}$ is the region of the orthogonality. Also, he gave some examples of orthogonal polynomials in $\nu$-variables satisfying a second order partial differential equation with two-parameter eigenvalues of the form (2.4). However, Bertran's results are not applicable if we do not know the orthogonalizing weight function and the region of orthogonality. We recall that there are many OPS's whose weight function is not known ([11, [15]).

Below, we show that our theorems can be applied to more examples which cannot be covered by Bertran's results.

Example 3.1. Consider the following partial differential equation:

$$
\left(x^{2}-1\right) u_{x x}+2 x y u_{x y}+\left(y^{2}-1\right) u_{y y}+g x u_{x}+g y u_{y}=n(n+g-1) u .
$$

The polynomial solutions are called the circle polynomials. Recently [12], we showed that the differential equation (3.4) has an OPS as solutions if $g \neq 1,0,-1, \cdots$. Let $\sigma^{(g)}$ be the orthogonalizing moment functional of the circle polynomials. Solving the equation (3.2) for $\sigma_{x}^{(g)}$ and $\sigma_{y}^{(g)}$, we have

$$
\left\{\begin{array}{l}
\left(\left(1-x^{2}-y^{2}\right) \sigma^{(g)}\right)_{x}=(1-g) x \sigma^{(g)}, \\
\left(\left(1-x^{2}-y^{2}\right) \sigma^{(g)}\right)_{y}=(1-g) y \sigma^{(g)} .
\end{array}\right.
$$

Thus by Theorem 2.4 and 2.6 $\left\{\mathbb{P}_{n}\right\}_{n=0}^{\infty}$ will satisfy three partial differential equations:

$$
\begin{gathered}
\left(1-x^{2}-y^{2}\right) \partial_{x}^{2} \mathbb{P}_{n}+(1-g) x \partial_{x} \mathbb{P}_{n}=\Lambda_{n} \mathbb{P}_{n}, \\
\left(1-x^{2}-y^{2}\right) \partial_{y}^{2} \mathbb{P}_{n}+(1-g) y \partial_{y} \mathbb{P}_{n}=\Gamma_{n} \mathbb{P}_{n}, \\
\left(1-x^{2}-y^{2}\right) \partial_{x}^{2} \mathbb{P}_{n}+\left(1-x^{2}-y^{2}\right) \partial_{y}^{2} \mathbb{P}_{n} \\
\quad+(1-g)\left[x \partial_{x} \mathbb{P}_{n}+y \partial_{y} \mathbb{P}_{n}\right]=\left(\Lambda_{n}+\Gamma_{n}\right) \mathbb{P}_{n},
\end{gathered}
$$

where $\Lambda_{n}$ and $\Gamma_{n}$ are constant matrices of order $(n+1) \times(n+1)$ given by

$$
\begin{aligned}
\Lambda_{n} & =\left(\begin{array}{ccccccc}
a_{n} & 0 & b_{n} & & & & \\
0 & a_{n-1} & 0 & & & & \\
& & & \ddots & & & \\
& & & & a_{2} & 0 & b_{2} \\
& & & & 0 & a_{1} & 0 \\
& & & & & 0 & 0
\end{array}\right), \\
\Gamma_{n} & =\left(\begin{array}{cccccccc}
0 & 0 & 0 & & & & & \\
0 & a_{1} & 0 & & & & \\
b_{2} & 0 & a_{2} & & & & \\
& & & \ddots & & & \\
& & & & 0 & a_{n-1} & 0 \\
& & & & b_{n} & 0 & a_{n}
\end{array}\right),
\end{aligned}
$$


where $a_{k}=-k(k+g-2)$ and $b_{k}=-k(k-1)$. Since the eigenvalues of $\Lambda_{n}$ and $\Gamma_{n}$ are all distinct, $\Lambda_{n}$ and $\Gamma_{n}$ are diagonalizable matrices. Thus by Theorem 2.5 . there is an $\operatorname{OB}\left\{\Phi_{n}\right\}_{n=0}^{\infty}$ (which in general is not monic) such that

$$
\left(1-x^{2}-y^{2}\right) \partial_{x}^{2} \phi_{n-k, k}+(1-g) x \partial_{x} \phi_{n-k, k}=a_{n-k} \phi_{n-k, k}(0 \leq k \leq n) .
$$

Similarly, there is an OB $\left\{\Psi_{n}\right\}_{n=0}^{\infty}$ (which in general is not monic) such that

$$
\left(1-x^{2}-y^{2}\right) \partial_{y}^{2} \psi_{n-k, k}+(1-g) y \partial_{y} \psi_{n-k, k}=a_{k} \psi_{n-k, k}(0 \leq k \leq n) .
$$

Furthermore, the partial derivatives $\left\{\mathbb{P}_{n}^{(x)}\right\}_{n=0}^{\infty}$ and $\left\{\mathbb{P}_{n}^{(y)}\right\}_{n=0}^{\infty}$ of $\left\{\mathbb{P}_{n}\right\}_{n=0}^{\infty}$ with respect to $x$ and $y$ are OB's relative to a moment functional $\left(1-x^{2}-y^{2}\right) \sigma^{(g)}$ (see [6]). In fact, we have

$$
\left(1-x^{2}-y^{2}\right) \sigma^{(g)}=\sigma^{(g+2)}
$$

Thus $\left\{\mathbb{P}_{n}^{(x)}\right\}_{n=0}^{\infty}$ or $\left\{\mathbb{P}_{n}^{(y)}\right\}_{n=0}^{\infty}$ is orthogonal relative to the moment functional $\sigma^{(g+2)}$ and satisfies the partial differential equation

$$
\left(x^{2}-1\right) u_{x x}+2 x y u_{x y}+\left(y^{2}-1\right) u_{y y}+(g+2) x u_{x}+(g+2) y u_{y}=n(n+g+1) u .
$$

Bertran dealt with the circle polynomials only for $g=3$. But he did not give the eigenvalues explicitly and did not notice that partial derivatives of $\left\{\mathbb{P}_{n}\right\}_{n=0}^{\infty}$ with respect to $x$ or $y$ are OB's relative to the weight function $w(x, y)=$ $H\left(1-x^{2}-y^{2}\right) d x d y$. We emphasize that our theorem has an advantage of being able to apply to the circle polynomials (with $g \neq 1,0,-1, \cdots$ ) and to prove the orthogonality of partial derivatives with respect to $x$ and $y$ of the normalization of the circle polynomials.

Remark 3.1. The matrix $\Omega_{n}=\Lambda_{n}+\Gamma_{n}$ in (3.7) has complex eigenvalues for some $n \geq 0$. Thus there is no real PS satisfying the partial differential equation with two-parameter eigenvalues

$$
\left(1-x^{2}-y^{2}\right) \partial_{x}^{2} v+\left(1-x^{2}-y^{2}\right) \partial_{y}^{2} v+(1-g)\left[x \partial_{x} v+y \partial_{y} v\right]=\omega_{n-k, k} v
$$

Remark 3.2. Schwartz (see [4] 16]) derived a pair of partial differential operators which have the circle polynomials as joint eigenfunctions. One of his partial differential operators is (3.7) with $g=2 \gamma+3$. He showed that the circle polynomials for $\gamma>-1$ are the unique solutions to the generalized Bochner problem [16]: Find all triples $\left(L_{1}, L_{2}, \mathcal{P}\right)$ where $\mathcal{P}$ is a family of polynomials, and $L_{i}(i=1,2)$ are second order linear partial differential operators such that $\phi_{1,0}(x, y):=x+i y \in \mathcal{P}$, and for every $\phi \in \mathcal{P}$

$$
\begin{aligned}
& \phi(1)=1, \bar{\phi} \in \mathcal{P}, \phi(\bar{z})=\bar{\phi}(z), \\
& L_{1} \phi=\phi_{x}(1) \phi, L_{2} \phi=\phi_{y}(1) \phi .
\end{aligned}
$$

Example 3.2. Consider the partial differential equation for the triangle polynomials

$$
\begin{aligned}
& \left(x^{2}-x\right) u_{x x}+2 x y u_{x y}+\left(y^{2}-y\right) u_{y y}+[(\alpha+\beta+\gamma+3) x-(\alpha+1)] u_{x} \\
& +[(\alpha+\beta+\gamma+3) y-(\beta+1)] u_{y}=\lambda_{n} u .
\end{aligned}
$$

The partial differential equation (3.8) has an OPS if $\alpha+1+n, \beta+1+n, \gamma+1+n$, $\alpha+\gamma+2+n, \beta+\gamma+2+n, \alpha+\beta+\gamma+3+n \neq 0$ for all $n \geq 0$ (see [12]). Then $\sigma$ satisfies the moment equations

$$
\left\{\begin{array}{l}
x y(x+y-1) \sigma_{x}=y[(\alpha+\gamma) x+\alpha y-\alpha] \sigma \\
x y(x+y-1) \sigma_{y}=x[\beta x+(\beta+\gamma) y-\beta] \sigma .
\end{array}\right.
$$


It is shown [7] that the common factors in (3.9) can be cancelled out and $\sigma$ satisfies

$$
\left\{\begin{array}{l}
{[x(x+y-1) \sigma]_{x}=[(\alpha+\gamma+2) x+(\alpha+1) y-(\alpha+1)] \sigma,} \\
{[y(x+y-1) \sigma]_{y}=[(\beta+1) x+(\beta+\gamma+2) y-(\beta+1)] \sigma .}
\end{array}\right.
$$

Then by Theorem 2.4, the monic triangle polynomials satisfy the partial differential equations

$$
\left\{\begin{array}{l}
x(x+y-1) \partial_{x}^{2} \mathbb{P}_{n}+[(\alpha+\gamma+2) x+(\alpha+1) y-(\alpha+1)] \partial_{x} \mathbb{P}_{n}=\Lambda_{n} \mathbb{P}_{n} \\
y(x+y-1) \partial_{y}^{2} \mathbb{P}_{n}+[(\beta+1) x+(\beta+\gamma+2) y-(\beta+1)] \partial_{y} \mathbb{P}_{n}=\Gamma_{n} \mathbb{P}_{n}
\end{array}\right.
$$

where $\Lambda_{n}$ and $\Gamma_{n}$ are constant matrices of order $(n+1) \times(n+1)$ given by

$$
\Lambda_{n}=\left(\begin{array}{ccccc}
\alpha_{n} & \beta_{n} & & & \\
0 & \alpha_{n-1} & & & \\
& & \ddots & & \\
& & & \alpha_{1} & \beta_{1} \\
& & & 0 & 0
\end{array}\right), \Gamma_{n}=\left(\begin{array}{ccccc}
0 & 0 & & & \\
\delta_{1} & \gamma_{1} & & & \\
& & \ddots & & \\
& & \gamma_{n-1} & 0 \\
& & \delta_{n} & \gamma_{n}
\end{array}\right)
$$

where $\alpha_{k}=k(k+\alpha+\gamma+1), \beta_{k}=k(k+\alpha), \gamma_{k}=k(k+\beta+\gamma+1)$ and $\delta_{k}=k(k+\beta)$. By Theorem 2.5, there is an OB $\left\{\Phi_{n}\right\}_{n=0}^{\infty}$ satisfying a second order partial differential equation with two-parameter eigenvalues for $n \geq 0$ and $0 \leq k \leq n$

$$
\begin{aligned}
x(x+y-1) \partial_{x}^{2} \phi_{n-k, k}+[ & (\alpha+\gamma+2) x+(\alpha+1) y \\
& -(\alpha+1)] \partial_{x} \phi_{n-k, k}=\alpha_{n-k} \phi_{n-k, k},
\end{aligned}
$$

since $\Lambda_{n}$ is diagonalizable if $\alpha+\gamma+2+n \neq 0$ for $n \geq 0$. Similarly, there is an OB $\left\{\Psi_{n}\right\}_{n=0}^{\infty}$ satisfying a second order partial differential equation with two-parameter eigenvalues for $n \geq 0$ and $0 \leq k \leq n$

$$
\begin{aligned}
y(x+y-1) \partial_{y}^{2} \psi_{n-k, k}+[ & (\beta+1) x+(\beta+\gamma+2) y \\
& -(\beta+1)] \partial_{y} \psi_{n-k, k}=\gamma_{k} \psi_{n-k, k},
\end{aligned}
$$

since $\Gamma_{n}$ is also diagonalizable if $\beta+\gamma+2+n \neq 0$ for $n \geq 0$.

The following example shows that our theorem improves Bertran's statement in the sense that our theorem can apply to an OPS relative to a moment functional $\sigma$ which satisfies a single equation of the form (2.1).

Example 3.3. Consider an OPS relative to a quasi-definite moment functional $\sigma$ satisfying the partial differential equation ([11], 15])

$$
3 y u_{x x}+2 u_{x y}-x u_{x}-y u_{y}+n u=0 .
$$

Then $\sigma$ satisfies

$$
\left\{\begin{array}{l}
(3 y \sigma)_{x}+\sigma_{y}=-x \sigma \\
\sigma_{x}=-y \sigma
\end{array}\right.
$$

Solving (3.11) for $\sigma_{x}$ and $\sigma_{y}$, we have

$$
\sigma_{x}=-y \sigma, \quad \sigma_{y}=\left(-x+3 y^{2}\right) \sigma .
$$

Note that (3.12) are neither of type considered by Bertran nor equations for a weight function. $\sigma$ is nothing but a moment functional and its integral representation is 
not known. Nevertheless, by Theorem $\left[2.4,\left\{\mathbb{P}_{n}\right\}_{n=0}^{\infty}\right.$ satisfy a system of partial differential equations

$$
\partial_{x}^{2} \mathbb{P}_{n}-y \partial_{x} \mathbb{P}_{n}=\Lambda_{n} \mathbb{P}_{n}
$$

where $\Lambda_{n}$ is a matrix of order $(n+1) \times(n+1)$ given by

$$
\Lambda_{n}=\left(\begin{array}{ccccccc}
0 & -n & 0 & & & & \\
0 & 0 & -n+1 & & & & \\
0 & 0 & 0 & & & & \\
& & & \ddots & & & \\
& & & & 0 & -2 & 0 \\
& & & & 0 & 0 & -1 \\
& & & & 0 & 0 & 0
\end{array}\right) .
$$

However, $\Lambda_{n}$ is not diagonalizable since the eigenvalues of $\Lambda_{n}$ are all 0 and the number of linearly independent eigenvectors is 1 . Thus there is no OB which satisfies the partial differential equation (3.10) and a partial differential equation of the form (2.4) simultaneously.

\section{ACKNOWLEDGMENTS}

The author thanks KOSEF (Korean Science and Engineering Foundation) for supporting his post doctoral experience in the USA. He especially thanks Professor Littlejohn at Utah State University for inviting him and discussing material in the research area.

\section{REFERENCES}

[1] W. A. Al-Salam and T. S. Chihara, Another characterization of the classical orthogonal polynomials, SIAM J. Math. Anal. 3 (1972), 65-70. MR 47:5320

[2] M. Bertran, Notes on orthogonal polynomials in v-variables, SIAM J. Math. Anal. 6(2) (1975), 250-257. MR 51:959

[3] S. Bochner, Über Sturm-Liouvillesche Polynomsysteme, Math. Z. 29 (1929), 65-72.

[4] W. C. Connett and A. L. Schwartz, Continuous 2-variable polynomial hypergroups. In (O. Gebuhrer W. C. Connett and A. L. Schwartz, editors), Applications of hypergroups and related measure algebras, pp. 89-109, Providence, R.I., 1995, American Mathematical Society. Contemporary Mathematics, 183. MR 96g:43006

[5] W. Hahn, Über die Jacobischen Polynom und zwei verwandte Polynomklassen, Math. Z. 39 (1935), 634-638.

[6] Y. J. Kim, K. H. Kwon and J. K. Lee, Orthogonal polynomials in two variables and second order partial differential equations, J. Comp. Appl. Math. 82 (1997), 239-260. MR 98m:33031

[7] Y. J. Kim, K. H. Kwon and J. K. Lee, Partial differential equations having orthogonal polynomial solutions, J. Comp. Appl. Math. 99 (1998), 239-253. MR 99j:35007

[8] M. A. Kowalski, Orthogonality and recursion formulas for polynomials in $n$ variables, SIAM J. Math. Anal. 13 (1982), 316-323. MR 83j:42022b

[9] M. A. Kowalski, Algebraic characterizations of orthogonality in the space of polynomials, Lecture Notes in Math. 1171 (1985), 101-110. MR 87j:42066

[10] H. L. Krall, On derivatives of orthogonal polynomials II, Bull. Amer. Math. Soc. 47 (1941), 261-264. MR 2:282

[11] H. L. Krall and I. M. Sheffer, Orthogonal polynomials in two variables, Ann. Mat. pura Appl. 4 (1967), 325-376. MR 37:4499

[12] K. H. Kwon, J. K. Lee and L. L. Littlejohn, Orthogonal polynomial eigenfunctions of second order partial differential equations, preprint.

[13] K. H. Kwon, J. K. Lee and B. H. Yoo, Characterizations of classical orthogonal polynomials, Results in Math. 24 (1993), 119-128. MR 94i:33011. 
[14] K. H. Kwon and L. L. Littlejohn, Classification of classical orthogonal polynomials, J. Korean Math. Soc. 34 No. 4 (1997), 973-1008. MR 99k:33028

[15] L. L. Littlejohn, Orthogonal polynomial solutions to ordinary and partial differential equations, Proc. 2nd Intern. Symp. Orthogonal polynomials and their applications, M. Alfaro et al. ed., Segovia (Spain), 1986, Lecture Notes in Math. Vol. 1329, Springer Verlag, Berlin, 1988, 98-124. MR 89j:33013

[16] A. L. Schwartz, Partial differential equations and bivariate orthogonal polynomials, preprint.

[17] N. Ja Sonine, Über die angenäherte Berechnung der bestimmten Integrale und über die dabei vorkommenden ganzen Functionen, Warsaw Univ. Izv 18 (1887), 1-76.

[18] Y. Xu, On multivariate orthogonal polynomials, SIAM J. Math. Anal. 24 (3) (1993), 783-794. MR 94i:42031

Department of Mathematics, Sunmoon University, Asan, Choongnam 336-840, Korea

E-mail address: jklee@omega.sunmoon.ac.kr 